BULL. AUSTRAL. MATH. SOC.

VOL. $24(1981), 321-325$.

\title{
A GENERALIZATION OF RADON'S THEOREM II
}

\author{
H. TVERBERg
}

A new proof is given of the following result:

Let $m$ and $d$ be positive integers, and let a set of $m d+m-d$ points be given in $d$-dimensional space. Then the set can be partitioned into $m$ sets such that the $m$ convex polytopes spanned by the sets have a non-empty intersection.

Let $n$-set mean a set of $n$ points in $R^{d}$. We shall say that an $n$-set is m-divisible if it can be divided into $m$ sets in such a way that the convex hulls of the $m$ sets have a non-empty intersection. In 1964, [6], I proved the following:

THEOREM. Any $(m(d+1)-d)-$ set is m-divisible.

The proof has been regarded as difficult and it is therefore a pleasure to be able to present a much simpler proof below. It is also a pleasure to acknowledge my debt to Imre Bárány, whose proof of Theorem 2.2 in [1] inspired the present one.

The proof is by induction on $m$. The case $m=1$ is trivial and so, assuming that the theorem holds for $m=k>0$, we are to prove that it holds for $m=k+1$. Put $K=(k+1)(d+1)-d$ and let a $K$-set $\Omega_{0}=\left\{p_{1}^{0}, \ldots, p_{K}^{0}\right\}$ be given. If the theorem is false for $\Omega_{0}$, then there is an $\varepsilon>0$ such that it is also false for any set $\Omega=\left\{p_{1}, \ldots, p_{K}\right\}$,

Received 28 April 1981. This research was done while the author was a Visiting Fellow in the Department of Mathematics, Research School of Physical Sciences, Institute of Advanced Studies, Australian National University. He was on sabbatical leave from the University of Bergen, supported by the Norwegian Research Council for Science and the Humanities. 
where $\left|p_{i}-p_{i}^{0}\right|<\varepsilon, i=1, \ldots, K$. We choose $\Omega$ strongly independent, as defined by Reay [4], and prove the theorem for $\Omega$, which suffices. Strong independence of $\Omega$ means that for each $t$ and for any $t$ affine subspaces $B_{1}, \ldots, B_{t}$ of $R^{d}$, spanned by pairwise disjoint subsets of $\Omega, \operatorname{dim}\left(B_{1} \cap \ldots \cap B_{t}\right)=\max \left(-1, \operatorname{dim} B_{1}+\ldots+\operatorname{dim} B_{t}-(t-1) d\right)$.

Consider a partition in $\Omega$, that is a partition of a subset of $\Omega$, consisting of disjoint non-empty sets $\Omega_{0}, \ldots, \Omega_{k}$, having the property that conv $\Omega_{1} \cap \ldots \cap$ conv $\Omega_{k} \neq \emptyset$. Such partitions exist by the case $m=k$ of the theorem. Choose such a partition for which the distance from conv $\Omega_{0}$ to conv $\Omega_{1} \cap \ldots \cap$ conv $\Omega_{k}$ is minimal. If this distance is zero, we are through, and so we assume it to be positive. A contradiction will now be obtained by showing that some other partition in $\Omega$ will make the distance considered smaller.

Let $q \in \operatorname{conv} \Omega_{0}$ and $r \in \operatorname{conv} \Omega_{1} \cap \ldots n$ conv $\Omega_{k}$ be a pair of points realizing the distance. By Carathéodory's theorem there is a simplex, with vertex set $\Omega_{0}^{\prime} \subseteq \Omega_{0}$, such that $q$ is in the relative interior of conv $\Omega_{0}^{\prime}$. Replacing $\Omega_{0}$ by $\Omega_{0}^{\prime}$, we may thus assume that $q$ is in the relative interior of conv $\Omega_{0}$. Similarly, we may assume that each conv $\Omega_{i}, i=1, \ldots, k$, is a simplex with $r$ in its relative interior. Putting $A_{i}=$ aff $\Omega_{i}$, the affine hull of $\Omega_{i}$, we then get $\operatorname{dim} A_{i}=\left|\Omega_{i}\right|-1, i=0, \ldots, k$. Furthermore, by the condition of strong independence,

$$
\begin{aligned}
\operatorname{dim}\left(A_{1} \cap \ldots \cap A_{k}\right)=\operatorname{dim} A_{1}+\ldots+\operatorname{dim} A_{k} & -(k-1) d \\
& =\left|\Omega_{1} \cup \ldots \cup \Omega_{d}\right|-k+d-k d .
\end{aligned}
$$

We now want to prove that $\Omega_{0} \cup \ldots \cup \Omega_{k}$ is a proper subset of $\Omega$, which will leave us some point $p_{j}$ to add to a suitable $\Omega_{i}$ so as to lower the distance in question. Consider the parallel hyperplanes ${ }_{q}$ through $q$ and $H_{r}$ through $r$, both orthogonal to $q-r$. The open 
slab between them clearly separates conv $\Omega_{0}$ from conv $\Omega_{1} \cap \ldots \cap$ conv $\Omega_{k}$. Furthermore $\Omega_{0} \subset H_{q}$, while conv $\Omega_{1} \cap \ldots n$ conv $\Omega_{k} \subset H_{p}$. The first of these inclusions holds true because $H_{q}$ is a supporting hyperplane of conv $\Omega_{0}$ in $q$ and $q$ is in the relative interior of the simplex conv $\Omega_{0}$. The second one holds because $r$ is in the relative interior of each simplex conv $\Omega_{1}, \ldots$, conv $\Omega_{k}$, so that some neighbourhood of $r$ in $A_{1} \cap \ldots \cap A_{k}$ is contained in conv $\Omega_{1} \cap \ldots \cap$ conv $\Omega_{k}$, and is thus supported by ${ }_{r}$ in $R$. (If it was not, it would meet the open slab mentioned above.) These two inclusions show that $A_{0} \subset H_{q}$ and $A_{1} \cap \ldots \cap A_{k} \subset H_{r}$, which, by the strong independence in $\Omega$ can happen only if

$$
\operatorname{dim} A_{0}+\operatorname{dim}\left(A_{1} \cap \ldots \cap A_{k}\right)<d .
$$

Thus

$$
\left|\Omega_{0}\right|-1+\left|\Omega_{1} \cup \ldots \cup \Omega_{k}\right|-k+d-k d<d,
$$

so that

$$
\left|\Omega_{0} \cup \ldots \cup \Omega_{k}\right|<k(d+1)+1=|\Omega| .
$$

It is no restriction to assume that $p_{1} \vDash \Omega_{0} \cup \ldots \cup \Omega_{k}$.

The easier case is when $p_{1}$ is in that open halfspace, bounded by $H_{q}$, in which $H_{r}$ lies. The segment $q p_{1}$ will then be in $\operatorname{conv}\left(\Omega_{0} \cup\left\{p_{1}\right\}\right)$ and for any point $q^{\prime}$ on it sufficiently near $q$, but not equal to $q$, we will have $\left|q^{\prime}-r\right|<|q-r|$. Thus the distance from $\operatorname{conv}\left(\Omega_{0} \cup\left\{p_{I}\right\}\right)$ to conv $\Omega_{I} \cap \ldots \cap$ conv $\Omega_{k}$ will be smaller than that from conv $\Omega_{0}$.

In the second, more difficult, case, $p_{1}$ is separated (weakly) from $H_{r}$ by $H_{q}$. We shall see that for some $i \in\{1, \ldots, k\}$ there is a ray from $r$, contained in $A_{1}, \ldots, A_{i-1}, A_{i+1}, \ldots, A_{k}$, which passes through the simplex $\operatorname{conv}\left(\Omega_{i} \cup\left\{p_{1}\right\}\right)$ and also lies in the halfspace bounded by $H_{r}$ 
and containing $H_{q}$. Any point $r^{\prime}(\neq r)$ on this ray, sufficiently near $r$, will then satisfy $\left|q-r^{\prime}\right|<|q-r|$ and also be in $\operatorname{conv} \Omega_{1} \cap \ldots \cap \operatorname{conv}\left(\Omega_{i} \cup\left\{p_{1}\right\}\right) \cap \ldots \cap \operatorname{conv} \Omega_{k}$, so that the desired contradiction will be obtained once again.

For the proof of the existence of the ray just mentioned, we introduce an affine coordinate system in which $r=(0, \ldots, 0), p_{1}=(1, \ldots, 1)$, $A_{1}$ is given by $x_{j}=0, j \in C_{1}, \ldots, A_{k}$ by $x_{j}=0, j \in C_{k}$. Here $c_{1}, \ldots, c_{k}$, form a partition in $\{1, \ldots, d\}$ (with possibly some empty parts). The disjointness of the $C_{i}$ is a consequence of the strong independence in $\Omega$. Furthermore $H_{r}$ is given by $a_{1} x_{1}+\ldots+a_{d} x_{d}=0$, where $a_{j}=0$ for $j \notin\left(C_{1} \cup \ldots \cup C_{k}\right)$, as we have seen that $A_{1} \cap \ldots \cap A_{k} \subset H_{r}$. The $a_{j}$ 's are normalized so that $p_{1}$ (which is not in $H_{r}$ ) lies in the hyperplane $a_{1} x_{1}+\ldots+a_{d} x_{d}=1$. Thus $H_{q}$ will have the equation $a_{1} x_{1}+\ldots+a_{d} x_{d}=a$, with $0<a \leq 1$.

Now consider the flat aff $\left(\Omega_{1} \cup\left\{p_{1}\right\}\right) \cap A_{2} \cap \ldots \cap A_{s}$. It is no restriction to assume that $C_{1}=\left\{1, \ldots,\left|C_{1}\right|\right\}$, $c_{2}=\left\{\left|C_{1}\right|+1, \ldots,\left|C_{1}\right|+\left|C_{2}\right|\right\}$ and so on. Then the point $p_{1}^{\prime}=(1, \ldots, 1,0, \ldots, 0)\left(\left|C_{1}\right| 1 ' s\right)$ is in the flat, as it equals $p_{1}+(0, \ldots, 0,-1, \ldots,-1)$. Furthermore $p_{1}^{\prime}$ and $p_{1}$ are in the same open halfspace of aff $\left(\Omega_{1} \cup\left\{p_{1}\right\}\right)$, bounded by $A_{1}$, as $(0, \ldots,-1, \ldots,-1)$ is in $A_{1}$. Hence the ray from $r$ through $p_{1}^{\prime}$ passes through the simplex $\operatorname{conv}\left(\Omega_{1} \cup\left\{p_{1}\right\}\right)$. It will also lie in the halfspace bounded by $H_{r}$ and containing $H_{q}$, provided $\left(a_{1}, \ldots, a_{d}\right) \cdot(1, \ldots, 1,0, \ldots, 0)=a_{1}+\ldots+a_{\left|C_{1}\right|}>0$. Thus, if $a_{1}+\ldots+a_{\left|C_{1}\right|}>0$, we have what we want. Similarly, we shall be satisfied with $a_{\left|C_{1}\right|+1}+\ldots+a_{\left|C_{1}\right|+\left|C_{2}\right|}>0$, and so on. But one of these equalities must hold, as $a_{1}+\ldots+a_{\left|c_{1}\right|+\ldots+\left|c_{k}\right|} \mid=1$. 
Doignon and Valette [2] have proved that our theorem remains valid in any affine space over an ordered division ring. The proof given above can be modified so as to show this. Finally I would like to call the reader's attention to the recent survey papers by Eckhoff [3] and Reay [5], which give a lot of information on Radon's theorem and related matters.

\section{References}

[1] Imre Bárány, "A generalization of Carathéodory's theorem", preprint.

[2] J.-P. Doignon and G. Valette, "Radon partitions and a new notion of independence in affine and projective spaces", Mathematika 24 (1977), 86-96.

[3] Jürgen Eckhoff, "Radon's theorem revisited", Contributions to geometry, 164-185 (Proc. Geom. Sympos., Siegen, 1978. Birkhäuser, Basel, 1979).

[4] John R. Reay, "An extension of Radon's theorem", IlZinois J. Math. 12 (1968), 184-189.

[5] J. Reay, "Open problems around Radon's theorem", Proceedings of the J. Clarence Karcher conference on convexity and related combinatorics (Marcel Dekker, to appear).

[6] H. Tverberg, "A generalization of Radon's theorem", J. London Math. Soc. 41 (1966), 123-128.

\footnotetext{
Department of Mathematics,

Institute of Advanced Studies,

Australian National University,

PO Box 4,

Canberra, ACT 2600,

Australia.

Permanent address:

Department of Mathematics,

University of Bergen,

5014 Bergen,

Norway.
} 